\title{
Diabetic Foot Evaluation in Family Medicine
}

\author{
Esad Alibasic ${ }^{1 *}$, Ahmet Tuzlak ${ }^{1}$, Farid Ljuca ${ }^{2}$, Emir Alibasic ${ }^{3}$ and Enisa Ramic ${ }^{4}$
}

${ }^{1}$ Department of Family Medicine, Primary Health Care Center Kalesija, Bosnia and Herzegovina

${ }^{2}$ Department of Physiology, Faculty of Medicine, University of Tuzla, Bosnia and Herzegovina

${ }^{3}$ Medical Faculty, University of Tuzla, Bosnia and Herzegovina

${ }^{4}$ Department of Family Medicine, Primary Health Care Center and Polyclinics Dr Mustafa Sehović, Tuzla, Bosnia and Herzegovina

\begin{abstract}
Introduction: Diabetes mellitus is characterized by the lack of glycemic control, which may cause damage to the small and large blood vessels and nerves, which could, among the other things, lead to changes in the foot. Most of diabetic foot complications that resulted in amputation begin with the formation of skin ulcers. The most important risk factors and predictors of diabetic ulcers are the presence of diabetic neuropathy and structural foot deformities, infections and peripheral occlusive arterial disease. Smoking, hypertension and hyperlipidemia also contribute to the increased prevalence.
\end{abstract}

Objective: To analyze the effectiveness of recommended interventions, conducted by family medicine team, for the prevention of diabetic foot ulcers.

Patients and methods: Data from the medical records of all patients with type 2 diabetes in the family medicine team 1 of Public Health Institution - Healthcare Center Kalesija were retroactively analyzed. Interventions that were implemented in the prevention of diabetic ulcers are as follows: screening for diabetic neuropathy using SemmesWeinstein's monofilament, regular and systematic examination of footwear and feet, education of patients and family members about proper hygiene and foot care, daily foot inspection by the patient, and other possible effective clinical interventions such as: optimizing the values of blood pressure, blood glucose, hyperlipidemia and smoking cessation.

Results: From total of 80 patients with type 2 diabetes, in $45(56 \%)$ was established peripheral neuropathy. From these 45 patients with neuropathy, in $40(88 \%)$ there has been no development of ulcers due to regular implementation of recommended interventions for prevention during each visit, and in 5 patients $(12.5 \%)$ the disease led to the development of diabetic ulcers. Of these 5 patients with diabetes that developed diabetic ulcer, $2(40 \%)$ underwent amputation due to poor communication and cooperation, irregular visits and the impossibility of implementing intervention and prevention measures.

Conclusion: The family medicine has a central role in the prevention and early diagnosis of diabetic foot complications. Patients with diabetes may benefit from preventive interventions, including screening for neuropathy, educating patients on every visit, wearing proper footwear, intensive care and supervision, as well as early identification of high risk for amputation and evaluation for surgical intervention. A multidisciplinary team approach is vital in the management of diabetic foot. Regular, careful and systematic review of the feet of patients with diabetes is one of the easiest, cheapest and most effective preventive intervention and measure for the prevention of diabetic foot complications.

Keywords: Preventive interventions; Diabetic foot; Neuropathy; Semmes-Weinstein's monofilament

\section{Introduction}

Diabetes affects $4.2 \%$ of the world population and is characterized by the lack of glycemic control, which may cause damage to the small and large blood vessels and nerves, which could, among the other things, lead to changes in the foot. Most of diabetic foot complications that resulted in amputation begin with the creation of skin ulcers. In people with diagnosed diabetes, the prevalence of foot ulcers is $4-10 \%$, the annual incidence is 1 to $4.1 \%$, and in the patient's lifetime the odd for incidence may be $25 \%$ [1].

Diabetic foot complications are the most common reason for nontraumatic lower limb amputations in the industrialized world and the most common reason for hospitalization of patients with diabetes [2]. The risk for lower extremity amputation is 15 to 46 times higher in patients with diabetes than in people without the desease [3]. The most important risk factors and predictors of diabetic ulcers are the presence of diabetic neuropathy and structural foot deformities, infections, and peripheral occlusive arterial disease. Smoking, hypertension and hyperlipidemia also contribute to the increased prevalence [4].
Neuropathy is most commonly associated with the development of diabetic foot ulcers, and the presence or the existance of peripheral arterial disease and infection can also lead to disruption and damage to the skin [5].

Preventive interventions carried out by a team of family medicine: In order to prevent later complications of diabetes, patients should be educated about the causes, recognition, progress, signs of deterioration and the possible consequences of late complications. Preventive

*Corresponding author: Esad Alibasic, Department of Family Medicine, Primary Health Care Center Kalesija, Bosnia and Herzegovina, Tel: 38770230914; E-mail: esad.alibasic@bih.net.ba

Received March 24, 2015; Accepted August 31, 2015; Published September 03 2015

Citation: Alibasic E, Tuzlak A, Ljuca F, Alibasic E, Ramic E (2015) Diabetic Foot Evaluation in Family Medicine. J Diabetes Metab 6: 600. doi:10.4172/21556156.1000600

Copyright: $\odot 2015$ Alibasic E, et al. This is an open-access article distributed unde the terms of the Creative Commons Attribution License, which permits unrestricted use, distribution, and reproduction in any medium, provided the original author and source are credited. 
interventions that nurse of an family medicine team regularly conducts are the following: Advising the patient to control the foot on daily basis (inaccessible parts of the foot can be seen in a mirror). Advising the patient if he notice swelling of the legs, change in the color of the foot skin, the formation of calluses, blisters or "corns", especially if he experience pain and / or fever, to immediately address the nurse. Advising patients to cut their toenails straight with their personal disinfected scissors. Warning patients to pay attention when choosing footwear (must be soft, wide enough, medium heels, with as few seams as possible), and that shoes must allow the skin to ventilate. Advising patients to wash their feet in the water that is warmed to body temperature and to use a mild and neutral soap (after washing, the feet should be well dried, especially between the toes). Also, pointing to the patients to pay close attention that the socks should be made of natural materials (cotton, silk, wool), and to avoid elastic garters. It is recommended that patients avoid wearing sandals or shoes on bare skin (without socks) - since it reduces the threshold of sensitivity to pain, which increases the possibility of damage of the skin and the occurrence of infections. Encourage the cessation of smoking and alcohol consumption. Warns the patients that they can not warm up their feet using hot water, heaters, or getting close to the fire. Prevention of diabetic foot ulcers family doctor begins by screening the patient for loss of protective sensation, which is the best feasible in primary care, in the office of family medicine, taking a brief medical history and testing by Semmes-Weinstein's monofilament. Specialist clinics may quantify neuropathy by biotensiometry and evaluate the vascular status of the lower extremity using Doppler ultrasound. One of the most important roles and tasks of the team of family medicine is to timely identify risk factors for lower-extremity amputations. The most important risk factors for amputation are as follows: lack of surface sensitivity due to peripheral neuropathy, arterial insufficiency, foot deformities, callus formation, autonomic neuropathy which causes skin dryness and reduces sweating and leads to the formation of fissures of the skin, limited joint mobility, obesity, impaired vision, poor control of blood sugar level, which leads to slower healing of wounds, inadequate footwear selection that causes skin damage, history of ulcers or amputation of the foot [6].

\section{Methodology}

\section{Screening for peripheral neuropathy}

Nylon monofilament test is a simple test to diagnose patient's risk for the formation of foot ulcers due to peripheral sensory neuropathy [7]. The test is unusual and there is present risk of ulcers formation if the patient can not feel the touch of an monofilament when pressed on foot with only so much pressure that can bend filament [8]. The patient is asked to say "yes" every time he feels the touch. The inability to feel filament on 4 out of 10 spots, is $97 \%$ sensitive and $83 \%$ specific method for identifying loss of protective sensation. Only calibrated nylon monofilament should be used in order to ensure optimum accuracy. Monofilaments can widely vary in accuracy due to differences in the length and diameter of the filaments. Also, due to the "memory" properties of nylon, monofilament requires two hours for recovery after 100 applications. Given that the skin sensitivity of patient will be tested at 20 different spots on both feet, after testing five patients the monofilament will lose it's accuracy. Therefore, it is necessary to have several monofilaments on hand to ensure the required accuracy. Further studies will examine when nylon monofilament requires complete replacement. Other clinical interventions that can possibly be effective in the prevention include: optimizing glycemic control modification of lifestyle (diet and exercise) [9] and pharmacotherapy,
$[10,11]$ optimizing blood pressure and lipid status, smoking cessation, debridement of calluses and certain types of preventive foot surgery.

\section{Prevention of the formation of diabetic ulcers}

Comprehensive history and systematic physical examination, especially the vascular status, as well as an examination of the patient's footwear conducted by the family doctor is essential, including a review of the regularity of walking and standing. Daily foot inspection by the patient or caregiver, if the patient does not see or has limited mobility, is crucial for proper foot care. Proper and regular foot care can delay and prevent complications that can cause the occurrence of diabetic foot.

Gentle cleansing with soap and water, followed by local application of hydrating agents, helps the skin to stay healthy.

\section{Evaluation and treatment of diabetic foot ulcers}

The classification system divides diabetic foot based on the risk of developing complications in six categories and is helping clinicians to identify critical parameters of diabetic foot dysfunction. There are several published risk categorizations and it is important that the team select and use the same categorization. Based on diagnostic parameters, Meggit and Wagner presented the clinical classification of diabetic foot syndrome, which is now used as a basis for treating this complex problem (Table 1).

The first three listed categories of the classification are the risk factors for foot ulcers, and the other three categories are risk factors for amputation. Risk classification, along with other findings from the history and physical examination, allows family physician to stratify patients based on risk and to determine the type of intervention. Depending on the assessment of risk categories, a family medicine physician intensifies and implements further preventive interventions, or suggesting appropriate subspecialists for the timely initiation of appropriate treatment for complications. Acceptance of a multidisciplinary team approach is vital in the management of diabetic foot $[12,13]$. This system quickly and accurately classifies patients with diabetes and guides the clinician in the selection of the most appropriate therapy for the prevention and therapeutic interventions [14].

\section{Objective}

To analyze the effectiveness of recommended interventions, conducted by family medicine team, for the prevention of diabetic foot ulcers.

\section{Patients and methods}

Data from the medical records of all patients with type 2 diabetes

0* No skin damage, foot deformities, sensory and motoric neuropathy Superficial ulceration

1* 1 A- Clean

1 B - Infected

Deep ulceration, penetrated to the tendons, joints or bones

2* 2 A - Clean

2 B - Infected

Deep abscess in planetary space, tendon shells, osteomyelitis and/or septic arthritis

3 A - Acute abscess

3B - Chronic abscess

Gangrene that affects one or more fingers or diffused front part of the foot

4* 4 A - Dry gangrene

$4 \mathrm{~B}$ - Moist gangrene

5* Gangrene that affects the entire foot

Table 1: The clinical classification of diabetic foot syndrome. 
Citation: Alibasic E, Tuzlak A, Ljuca F, Alibasic E, Ramic E (2015) Diabetic Foot Evaluation in Family Medicine. J Diabetes Metab 6: 600. doi:10.4172/2155-6156.1000600

Page 3 of 3

in the family medicine team 1 of Public Health Institution - Healthcare Center Kalesija were retroactively analyzed. Interventions that were implemented in the prevention of diabetic ulcers are as follows: screening for diabetic neuropathy using Semmes-Weinstein's monofilament, regular and systematic examination of footwear and feet, education of patients and family members about proper hygiene and foot care, daily foot inspection by the patient, and other possible effective clinical interventions such as optimizing the values of blood pressure, blood glucose, hyperlipidemia and smoking cessation.

\section{Results}

From total of 80 patients with type 2 diabetes, in 45 (56\%) was established peripheral neuropathy (Figure 1). From these 45 patients with neuropathy, in $40(87.5 \%)$ there has been no development of ulcers due to regular implementation of recommended interventions for prevention during each visit, and in 5 patients (12.5\%) the disease led to the development of diabetic ulcers. Of these 5 patients with diabetes that developed diabetic ulcer, 2 (40\%) underwent amputation due to poor communication and cooperation, irregular visits and the impossibility of implementing intervention and prevention measures (Table 2).

\section{Discussion}

There is evidence to support the opinion that screening all patients with diabetes for neuropathy can identify those who are at risk for developing diabetic ulcers [15]. In our study, in 56\% of patients with diabetes a peripheral diabetic neuropathy was established, which is consistent with the results of similar surveys and published studies [16]. In industrialized countries, diabetes is the leading cause for nontraumatic lower extremity amputations, and in $15 \%$ of patients with diabetes the development of foot ulcers occurs during disease, which is consistent with our results. Some studies have shown that $85 \%$ of diabetic ulcers end up with amputation of the lower extremities [17]. In our study only $40 \%$ of patients with ulcer underwent the amputation, which increases the importance of the implementation of preventive interventions for diabetic foot.

\section{Conclusion}

The family medicine has a central role in the prevention and early diagnosis of diabetic foot complications. Patients with diabetes may benefit from preventive interventions, including screening for neuropathy, educating patients during every visit, wearing proper footwear, intensive care and supervision, as well as early identification of high risk for amputation and evaluation for surgical intervention. A multidisciplinary team approach is vital in the management of diabetic foot. Regular, careful and systematic review of the feet of patients with diabetes is one of the easiest, cheapest and most effective

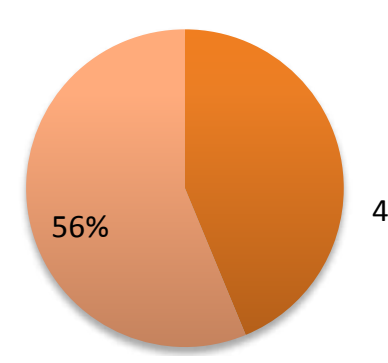

Without neuropathy With neuropathy

Figure 1: The prevalence of peripheral neuropathy in patients with type 2 diabetes.

\begin{tabular}{|l|c|c|c|c|c|}
\hline $\begin{array}{c}\text { Complications } \\
\text { 80 patients } \\
\text { with diabetes } \\
\text { type 2, with } \\
\text { recommended } \\
\text { preventive } \\
\text { interventions: }\end{array}$ & Neuropathy & $\begin{array}{c}\text { Neuropathy, } \\
\text { foot ulcer } \\
\text { developed }\end{array}$ & $\begin{array}{c}\text { Neuropathy, } \\
\text { foot ulcer not } \\
\text { developed }\end{array}$ & $\begin{array}{c}\text { Neuropathy, } \\
\text { foot ulcer } \\
\text { developed, } \\
\text { foot } \\
\text { amputated }\end{array}$ & $\begin{array}{c}\text { Neuropathy, } \\
\text { foot ulcer } \\
\text { developed, } \\
\text { foot not } \\
\text { amputated }\end{array}$ \\
$\begin{array}{c}\text { Number of } \\
\text { patients with } \\
\text { complications }\end{array}$ & $45 / 80$ & $5 / 45$ & $40 / 45$ & $2 / 5$ & $3 / 5$ \\
\hline Percentage & $56 \%$ & $12,5 \%$ & $87,5 \%$ & $40 \%$ & $60 \%$ \\
\hline
\end{tabular}

Table 2: The effect of preventive interventions on diabetic foot ulcers and amputation.

preventive interventions and measures for the prevention of diabetic foot complications.

\section{References}

1. Singh N, Armstrong DG, Lipsky BA (2005) Preventing foot ulcers in patients with diabetes. JAMA 293: 217-228.

2. Reiber GE, Pecoraro RE, Koepsell TD (1992) Risk factors for amputation in patients with diabetes mellitus. A case-control study. Ann Intern Med 117: 97105.

3. Armstrong DG, Lavery LA, Quebedeaux TL, Walker SC (1997) Surgical morbidity and the risk of amputation due to infected puncture wounds in diabetic versus nondiabetic adults. South Med J 90: 384-389.

4. Orsted HL, Searles GE, Trowell H, Shapera L, Miller P, et al. (2007) Best practice recommendations for the prevention, diagnosis, and treatment of diabetic foot ulcers: update 2006. Adv Skin Wound Care 20: 655-669.

5. RNAO (Registered Nurses' Association of Ontario) guidelines provide operational and educational recommendations that support and are the backbone to clinical practice recommendations.

6. Sibbald RG, Ayello EA, Alavi A, Ostrow B, Lowe J, et al. (2012) Screening for the high-risk diabetic foot: a 60-second tool (2012). Adv Skin Wound Care 25: 465-476.

7. Birke JA, Sims DS (1986) Plantar sensory threshold in the ulcerative foot. Lepr Rev 57: 261-267.

8. Armstrong DG, Lavery LA, Vela SA, Quebedeaux TL, Fleischli JG. Choosing a practical screening instrument to identify patients at risk for diabetic foot ulceration. Arch Intern Med 158: 289-292.

9. Alibasic E, Ramic E, Alic A (2013) Prevention of diabetes in family medicine Mater Sociomed 25: 80-82.

10. PoljakoviÄ $\ddagger D$, Hajder M (2003) [The role of insulin reserve in choice of therapy in type 2 diabetes mellitus]. Med Arh 57: 131-136.

11. PoljakoviÄ $\ddagger$ D, Hajder M (2002) [Treatment of type 2 diabetes mellitus based on parameters of B-cell functional reserve]. Med Arh 56: 195-200.

12. Mcewen L, Ylitalo K, Wrobel J, Herman W (2014) Foot Complications and Mortality: Results from Translating Research Into Action for Diabetes (TRIAD). American Diabetes Association (ADA) 74th Scientific Sessions, San Francisco California.

13. Bruntz M, Berg C, Garcia J, Price C (2014) A Diabetic Limb Salvage Program in an At-Risk Diabetic Population-Can It Be Successful? American Diabetes Association (ADA) 74th Scientific Sessions, San Francisco, California.

14. Armstrong DG, Lavery LA, Harkless LB (1998) Validation of a diabetic wound classification system. The contribution of depth, infection, and ischemia to risk of amputation. Diabetes Care 21: 855-859.

15. Singh N, Armstrong DG, Lipsky BA (2005) Preventing foot ulcers in patients with diabetes. JAMA 293: 217-228.

16. Harati Y (1994) Diabetic peripheral neuropathy. In: Kominsky SJ, ed. Medical and surgical management of the diabetic foot. St. Louis: Mosby, 73-85.

17. United States National Diabetes Advisory Board (1987) The national long range plan to combat diabetes. Bethesda, Md.: U.S. Department of Health and Human Services, Public Health Service, National Institutes of Health, 88-1587. 